\title{
Human cytomegalovirus protein pUL36: A dual cell death pathway inhibitor
}

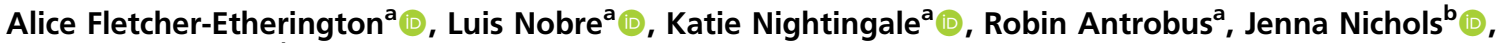

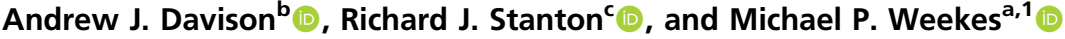 \\ ${ }^{a}$ Cambridge Institute for Medical Research, University of Cambridge, Cambridge CB2 OXY, United Kingdom; ${ }^{b}$ Medical Research Council-University of \\ Glasgow Centre for Virus Research, Glasgow G61 1QH, United Kingdom; and 'Division of Infection and Immunity, Cardiff University School of Medicine, \\ Cardiff CF14 4XN, United Kingdom
}

Edited by Thomas Shenk, Princeton University, Princeton, NJ, and approved June 25, 2020 (received for review February 3, 2020)

Human cytomegalovirus (HCMV) is an important human pathogen and a paradigm of intrinsic, innate, and adaptive viral immune evasion. Here, we employed multiplexed tandem mass tag-based proteomics to characterize host proteins targeted for degradation late during HCMV infection. This approach revealed that mixed lineage kinase domain-like protein (MLKL), a key terminal mediator of cellular necroptosis, was rapidly and persistently degraded by the minimally passaged HCMV strain Merlin but not the extensively passaged strain AD169. The strain Merlin viral inhibitor of apoptosis pUL36 was necessary and sufficient both to degrade MLKL and to inhibit necroptosis. Furthermore, mutation of pUL36 Cys $^{131}$ abrogated MLKL degradation and restored necroptosis. As the same residue is also required for pUL36-mediated inhibition of apoptosis by preventing proteolytic activation of procaspase-8, we define pUL36 as a multifunctional inhibitor of both apoptotic and necroptotic cell death.

human cytomegalovirus | cell death | necroptosis | protein degradation | MLKL

$\mathbf{H}$ uman cytomegalovirus (HCMV) infects the majority of the world's population, with a seroprevalence of 60 to $80 \%$ in Western Europe and the United States, and up to $100 \%$ in developing countries $(1,2)$. Following primary infection, a latent infection is established that persists lifelong. Reactivation from latency to productive infection in immunocompromised people can lead to significant morbidity or mortality, in addition to allograft rejection in transplant recipients $(3,4)$. Furthermore, HCMV is the commonest infectious cause of congenital disease, affecting $\sim 1 / 200$ pregnancies (5).

Only four drugs are approved by the FDA to treat HCMV, and all are associated with significant toxicity and the threat of drug resistance $(2,6)$. Small molecule-based disruption of interactions between antiviral host proteins and their viral antagonists could facilitate endogenous inhibition of viral replication $(7,8)$. Identification and detailed characterization of such interactions thus has important implications for the development of novel anti-HCMV therapies.

HCMV encodes 170 canonical protein-coding genes (2). A substantial number of additional, noncanonical open reading frames (ORFs) that potentially encode proteins have been identified by ribosomal footprinting and proteomics $(9,10)$ During productive infection in vitro, expression of HCMV genes is conventionally divided into immediate-early, early, and late phases during a $296-\mathrm{h}$ lytic replication cycle. Recently, five temporal classes of viral protein expression (Tp1 to Tp5) have been defined by measuring viral protein profiles over the whole course of infection (11).

Hijacking of the ubiquitin-proteasome system (UPS) to degrade host proteins is ubiquitous across many viral families (12). The proteins degraded by viruses are typically detrimental to viral replication, and can include antiviral restriction factors or components of viral sensing pathways, activating immune cell ligands, and elements of cell death pathways $(13,14)$. We have shown previously that $>900$ host proteins are down-regulated more than threefold over the course of HCMV infection, with 133 proteins degraded during the early phase, of which $89 \%$ are targeted to the proteasome. These data led directly to the identification of candidate natural killer (NK) cell ligands and identified helicase-like transcription factor (HLTF) as an antiviral restriction factor $(10,11)$. However, it is not yet known which proteins are degraded later during infection, or throughout a whole infection time course. Furthermore, it is unclear whether or how HCMV inhibits necroptotic cell death pathways, which represent a key defense against viral spread within an infected host (15).

Activation of death receptors such as TRAILR1/2, TNFR1, and Fas leads to apoptotic cell death via an activating cleavage of procaspase-8 (16). In the presence of caspase inhibition or limiting levels of ATP, extrinsic apoptosis shifts toward the necroptotic pathway. This is dependent on an interaction between Receptor Interacting Serine/Threonine Kinases 1 and 3 (RIP1/3) through their homotypic interaction motif (RHIM) domains (17). Necroptosis can also be activated by cytoplasmic sensing of murine cytomegalovirus (MCMV) DNA by Z-DNA-binding protein 1 (ZBP1), or ligation of Toll-like receptors 3 or 4 (TLR3/4),

\section{Significance}

Cell death is a key defense against viral infection, preventing spread from infected to uninfected cells. Correspondingly, certain viruses encode inhibitors of apoptotic and necroptotic cell death pathways in order to facilitate their persistence. Human cytomegalovirus (HCMV) is an important human pathogen that can block apoptosis, but hitherto it has been unclear whether or how the virus blocks necroptosis. Here, we used a proteomic screen to identify human proteins targeted for destruction by HCMV, finding that the key necroptosis mediator MLKL is degraded throughout infection. MLKL is targeted for degradation by HCMV protein pUL36, which is also instrumental in inhibiting apoptosis. Thus, pUL36 is a dual cell death pathway inhibitor, and may represent an important therapeutic target.

Author contributions: A.F.-E. and M.P.W. designed research; A.F.-E., L.N., K.N., R.A., and J.N. performed research; A.J.D. and R.J.S. contributed new reagents/analytic tools; A.F.-E., A.J.D., and M.P.W. analyzed data; A.F.-E. and M.P.W. wrote the paper; and L.N., K.N., A.J.D., and R.J.S. edited the paper.

The authors declare no competing interest.

This article is a PNAS Direct Submission.

This open access article is distributed under Creative Commons Attribution License 4.0 (CC BY).

Data deposition: The mass spectrometry proteomics data have been deposited to the ProteomeXchange Consortium (www.proteomexchange.org/) via the PRIDE partner repository with the dataset identifier PXD017279.

${ }^{1}$ To whom correspondence may be addressed. Email: mpw1001@cam.ac.uk.

This article contains supporting information online at https://www.pnas.org/lookup/suppl/ doi:10.1073/pnas.2001887117/-/DCSupplemental.

First published July 20, 2020. 
which activate RIP3 through alternative RHIM domain-containing adaptors (17) (Fig. 1). All three pathways converge with RIP3dependent phosphorylation and activation of the pseudokinase MLKL, which transitions from an inactive monomer to a necroptotic oligomer. The oligomer translocates to and disrupts the plasma membrane, leading to cell swelling and loss of plasma membrane integrity, although the exact mechanism of membrane disruption is unclear (18-20).

Here, a systematic examination of protein degradation at $48 \mathrm{~h}$ of HCMV infection determined that degradation of MLKL was sustained throughout early and late infection. The data showed that MLKL was degraded by the minimally passaged Merlin strain of HCMV, but not by the highly passaged laboratoryadapted strain AD169. The strain Merlin UL36-encoded viral inhibitor of caspase-8 activation (vICA/pUL36), which is known to function as a potent inhibitor of apoptosis, interacted with MLKL and was necessary and sufficient both to degrade MLKL and to inhibit TNF $\alpha$-stimulated necroptosis. Whereas HCMV strain AD169 sensitized cells to necroptosis, strain Merlin prevented this sensitization. pUL36 is thus a multifunctional cell death inhibitor capable of inhibiting both apoptotic and necroptotic pathways.

\section{Results}

Host Proteins Targeted for Degradation at $\mathbf{4 8}$ h of HCMV Infection. We applied the proteasomal inhibitor MG132 to build a global picture of proteins degraded late during HCMV infection. TERT-immortalized primary human fetal foreskin fibroblasts (HFFF-TERTs) were infected with HCMV strain Merlin at a multiplicity of infection (MOI) of 10 for $48 \mathrm{~h}$, with application of MG132 (or the equivalent amount of dimethyl sulfoxide (DMSO) as a control) for the final $12 \mathrm{~h}$ of infection (Fig. 2A). MG132 is known to inhibit calpains and lysosomal cathepsins in addition to the proteasome (10) and was used to generate a comprehensive list of proteins targeted for degradation by HCMV rather than to identify proteins specifically degraded by the proteasome. A total of 8,476 human and 186 viral proteins

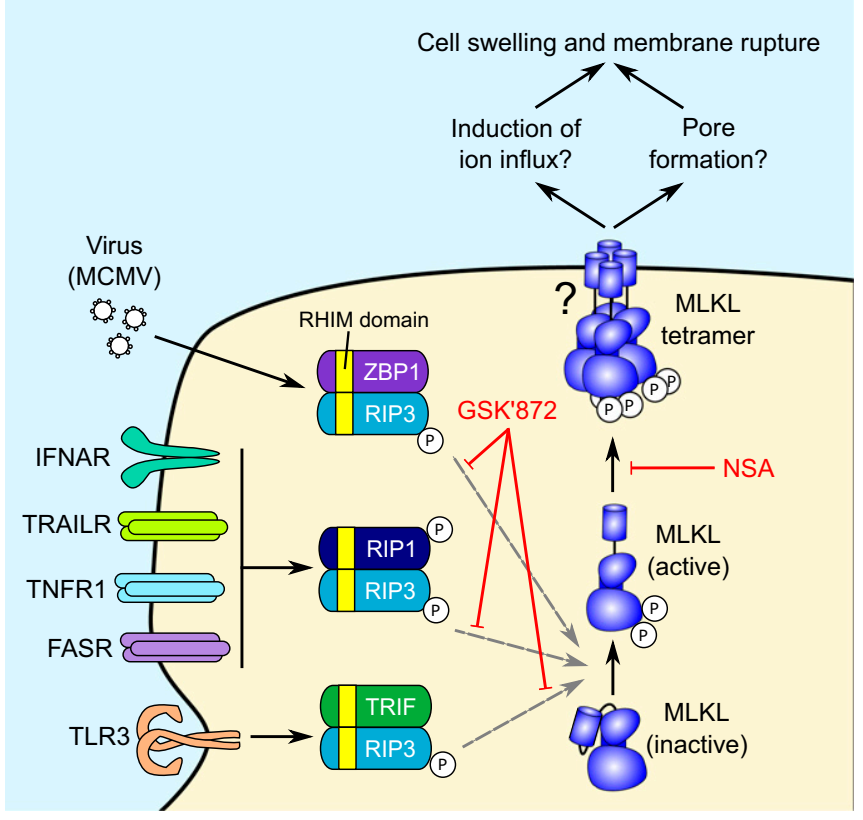

Fig. 1. The necroptosis pathway and mechanism of action of small molecule necroptosis inhibitors. Two inhibitors of the necroptotic pathway were utilized in this study: GSK' 872 , which inhibits RIP3 kinase activity $(21,22)$, and necrosulfonamide (NSA), which inhibits downstream effector functions of MLKL via covalent reaction with human $\operatorname{MLKL}$ residue $\operatorname{Cys}^{86}(19,23)$. were quantified across two biological replicates (Dataset S1, which includes an interactive "Plotter"). Two ratios and associated significance values were calculated for each protein: 1) virus:mock infection and 2) virus + MG132:virus infection (Fig. 2B). Fifty-two proteins met high-confidence criteria for degradation, with a fold down-regulation and rescue of $>2$ and $P<0.05$ for both ratios.

From a comparison with our previous study of HCMVinduced protein degradation between 2 and $24 \mathrm{~h}$ (10), seven proteins were degraded with high confidence throughout early and late infection (Fig. $2 C$ and $D$ ). These proteins included HLTF and Anaphase Promoting Complex subunits 1 and 5 (ANAPC1/5), whose degradation by HCMV has been well characterized $(10,25)$. The effector of necroptosis MLKL was the most significantly down-regulated protein at $48 \mathrm{~h}$ of HCMV infection and was among proteins most significantly rescued by addition of MG132 (Fig. 2B). In comparison, 37 proteins degraded at $48 \mathrm{~h}$ did not score highly for degradation at early time points (10) (Fig. $2 C$ and $E$ and SI Appendix, Fig. S1A).

Degradation of MLKL Is Mediated by Immediate Early Protein pUL36. We previously took a systematic approach to identifying the viral proteins responsible for the degradation of host factors, employing a panel of recombinant viruses deleted for various blocks of HCMV genes that are nonessential for replication in vitro (10). These viruses included strain AD169, a highly passaged, laboratory-adapted strain that contains a deletion in the $\mathrm{U}_{\mathrm{L}} / b^{\prime}$ region (encoding 20 canonical genes, UL133UL150A), frameshifts in RL5A, RL13, and UL131A, and a nonsynonymous substitution in UL36 that inactivates the ability of pUL36 to bind procaspase- 8 and inhibit apoptosis $(26,27)$. MLKL was down-regulated by strain Merlin viruses but not by strain AD169, and this was confirmed by immunoblotting (Fig. $3 A$ and $B$ ). This indicated that one or more differences in AD169 abrogate virus-mediated MLKL degradation. To identify viral factors interacting with MLKL, a SILAC immunoprecipitation (IP) was performed in HCMV strain Merlin-infected HFFF-TERTs stably expressing MLKL tagged with HA at the $\mathrm{C}$ terminus. pUL36 coprecipitated with MLKL, and this was confirmed by a reciprocal IP (Fig. $3 C$ and $D$ and Dataset S2). This interaction was further supported by an immunofluorescence study showing cytoplasmic colocalization between MLKLHA and pUL36 in stably expressing HFFF-TERTs (SI Appendix, Fig. S2A). To determine whether pUL36 interacts with inactive, unphosphorylated MLKL or active, phosphorylated MLKL we researched the pUL36-V5 SILAC IP data (Fig. 3D) using a variable phosphomodification. All identified MLKL peptides were unphosphorylated, and encompassed all known sites of activating phosphomodifications (SI Appendix, Fig. S3). This suggests that pUL36 interacts with unphosphorylated MLKL but does not exclude an additional interaction with phosphorylated MLKL.

A series of HFFF-TERT cell lines stably expressing strain Merlin UL36 or each of the individual genes in the UL133UL150A region were next screened to determine whether any other viral protein contributed to MLKL degradation. Expression of UL36 was sufficient to reduce the level of MLKL by 3.7fold (Fig. $3 E$ and SI Appendix, Fig. S2 $B-D$ ). MLKL expression was not modulated more than 2-fold by any other proteins in the $\mathrm{U}_{\mathrm{L}} / b^{\prime}$ region. Despite substantial variation in the level of expression of some of the viral proteins, this did not correlate with relative MLKL abundance (SI Appendix, Fig. S2D). pUL36 could be detected from 6 hours postinfection (hpi), and was expressed with Tp2 (temporal protein profile 2) kinetics, matching the kinetics of MLKL degradation (11) (Fig. $3 F$ ). Finally, infection with strain AD169 or two independent strain Merlin UL36 deletion mutants rescued MLKL, suggesting that pUL36 was necessary as well as sufficient for MLKL degradation (Fig. $3 G$ ). 


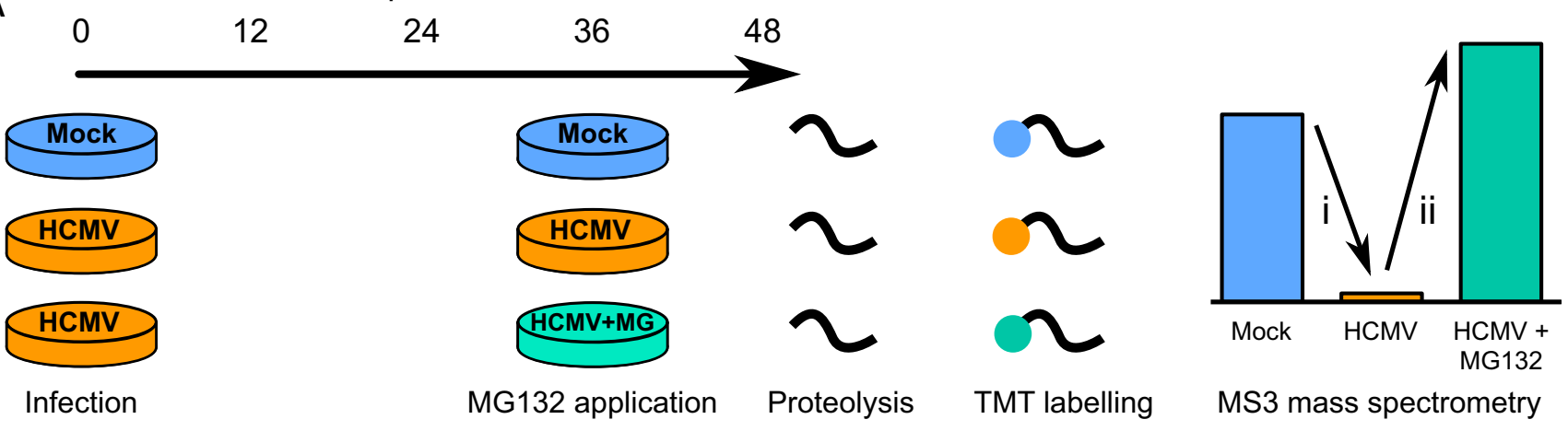

B

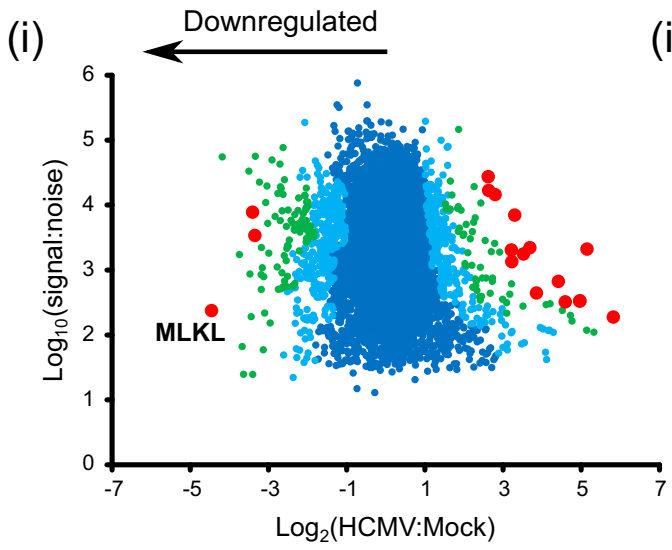

(ii)

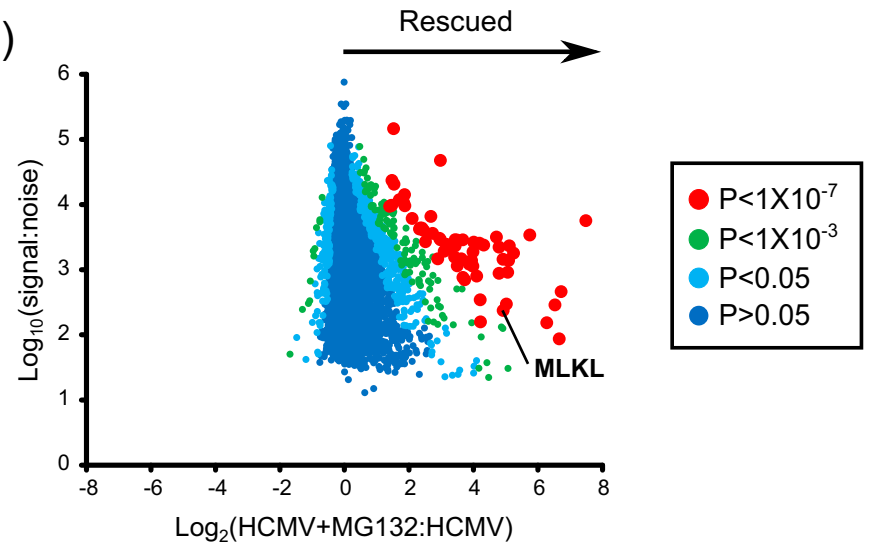

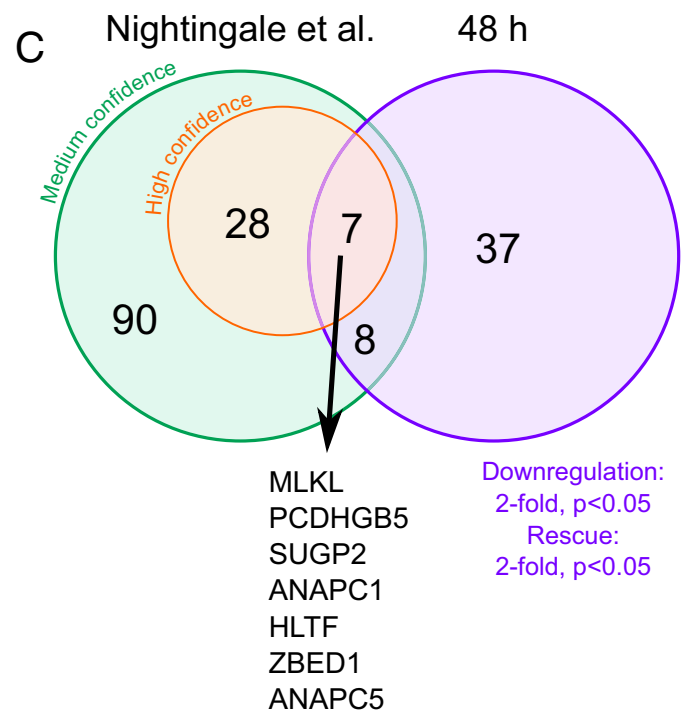
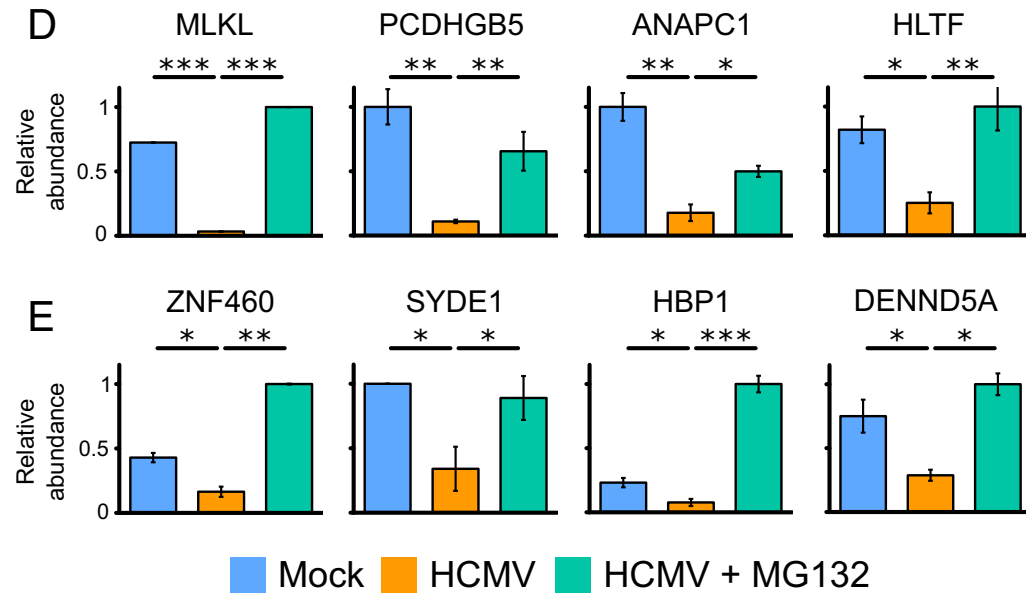

HBP1

DENND5A

Fig. 2. Identification of proteins targeted for degradation by HCMV at $48 \mathrm{hpi}$. (A) Schematic of the experimental method, conducted in biological duplicate. Cellular lysates from the second 48 -h biological replicate were analyzed simultaneously with residual lysates from the 12-h degradation screen in the Nightingale et al. (10) study, facilitating a direct comparison (SI Appendix, Fig. S1A). Peptides from each sample were labeled with tandem mass tags and analyzed by MS3 mass spectrometry. $(B)$ Scatterplots of human proteins quantified at $48 \mathrm{hpi}$ in one or both replicates, showing averaged ratios. $P$ values were estimated using significance $B$ values, then corrected for multiple hypothesis testing (24). K-means clustering suggested there were at least nine different patterns of protein expression across the samples (Dataset S1C and SI Appendix, Fig. S1B). (C) Overlap between early degradation data from the Nightingale et al. (10) study (using either stringent or sensitive statistical criteria) and $48 \mathrm{~h}$ degradation data presented in this study. (D) Examples of the seven proteins degraded with high confidence both early and late during infection. MLKL was only quantified in one replicate. Error bars: range. $P$ values were calculated as described in $B . * P<0.05, * * P<0.001, * * * P<1 \times 10^{-7}$. Additional proteins were likely to have been degraded both early and late during infection (Dataset $\mathrm{S} 1$ and SI Appendix, Fig. S1A), but did not pass the stringent filtering criteria used at one or other of the time points. (E) Examples of the 37 proteins degraded at $48 \mathrm{~h}$ that were not confidently degraded at early time points. These comprised: 1) proteins not degraded at early time points (shown in this figure), 2) proteins that were insufficiently degraded at early time points to pass filtering criteria, and 3) proteins not quantified in the Nightingale et al. (10) study. Additional examples and the corresponding $12 \mathrm{~h}$ data are shown in SI Appendix, Fig. S1A. 
A

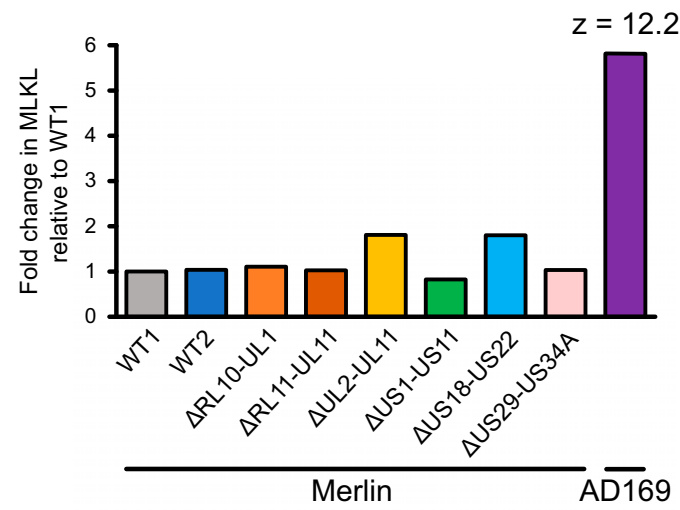

B

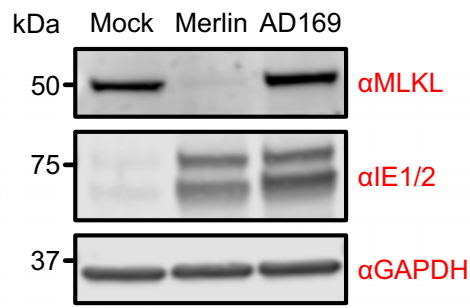

C
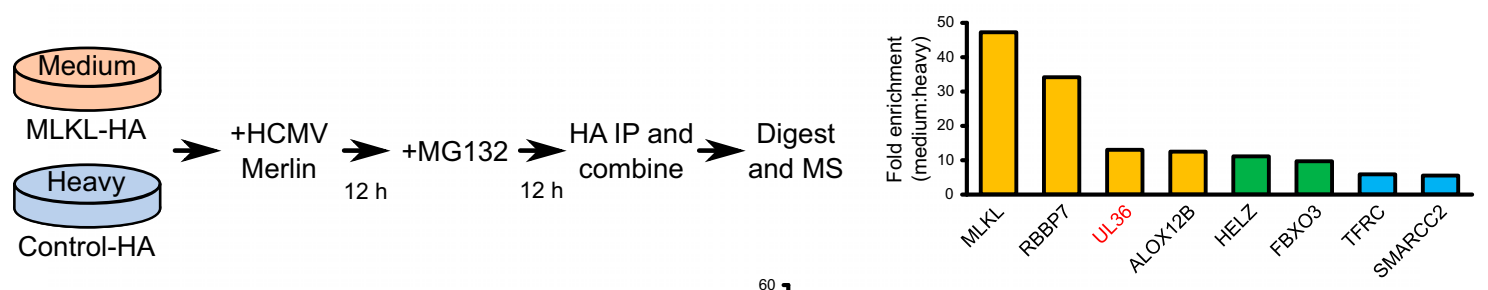

D
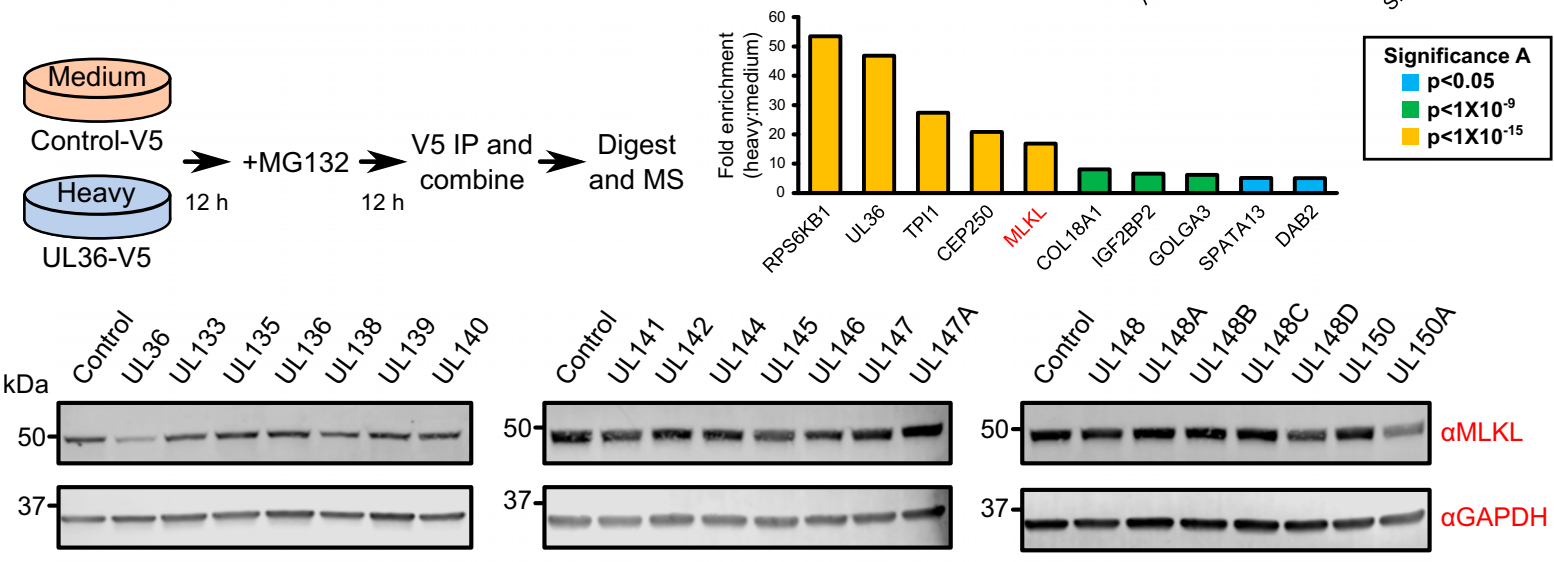

E

$\mathrm{F}$

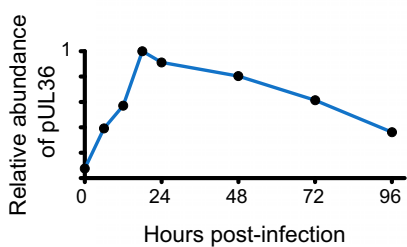

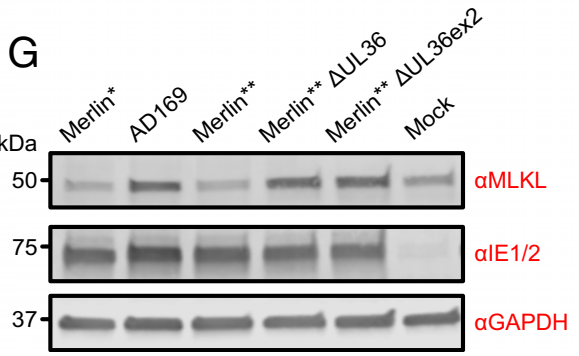

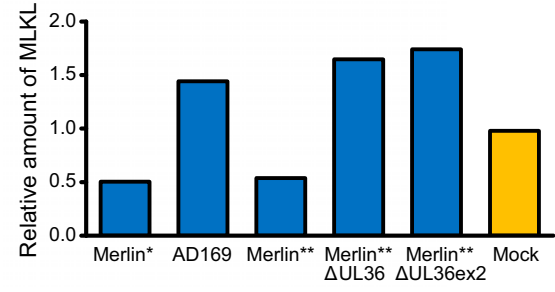

Fig. 3. HCMV strain Merlin pUL36 is necessary and sufficient to degrade MLKL. (A) Relative abundance of MLKL from a proteomic screen of $>250$ proteins published in the Nightingale et al. (10) study. HFFF-TERTs were infected with strain Merlin (WT1), WT1 that lacked UL16 and UL18 (WT2), one of six blockdeletion viruses derived from WT1 or WT2, or strain AD169 (MOI $=10,72 \mathrm{~h}$ infection). A $z$ score of $>5$ was considered significant. (B) Immunoblot confirming that MLKL is down-regulated by strain Merlin but not by strain AD169 (MOI $=5,48 \mathrm{~h}$ infection, cells lysed in RIPA buffer). MLKL was similarly down-regulated in cells lysed in 2\% SDS (SI Appendix, Fig. S2E). This was consistent with the observed MLKL down-regulation in cells lysed with $6 \mathrm{M}$ guanidine for proteomic experiments (Fig. 2) and indicated that MLKL was likely degraded as opposed to being translocated to RIPA-insoluble membrane-associated complexes. (C) SILAC immunoprecipitation of C-terminally HA-tagged MLKL or control in the presence of strain Merlin infection ( $\mathrm{MOI}=3,24 \mathrm{~h}$ infection in the presence of 10 $\mu \mathrm{M}$ MG132 for the final $12 \mathrm{~h}$ ). Proteins enriched more than fivefold are shown. $P$ values were estimated using the method of significance $A$ and corrected for multiple hypothesis testing (24). (D) SILAC immunoprecipitation of C-terminally V5-tagged pUL36 or control in the presence of $10 \mu \mathrm{M}$ MG132 for $12 \mathrm{~h}$. Proteins that were enriched more than fivefold are shown, and $P$ values were estimated as described in $C$. (E) Immunoblot showing that pUL36 is sufficient to downregulate MLKL. A series of HFFF-TERT cell lines stably expressing genes in the UL133-150A region were lysed in RIPA buffer and analyzed by immunoblotting. Confirmation of viral protein expression was achieved by immunoblotting or mass spectrometry (28) (Dataset S3D and SI Appendix, Fig. S2B), except for pUL136, which was not detected by either method. MLKL was similarly down-regulated by pUL36 in cells lysed in $2 \%$ SDS (SI Appendix, Fig. S2F, as shown during HCMV infection in SI Appendix, Fig. S2E). (F) Temporal profile of strain Merlin pUL36 in whole-cell lysates harvested at different times over the whole course of infection, from the Weekes et al. (11) study. (G) Immunoblot showing that pUL36 is necessary for down-regulation of MLKL (MOI $=5,24 \mathrm{~h}$ infection). Cells were infected with WT strain Merlin (Merlin*), a version of Merlin in which intact genes UL128 and RL13 are under tet regulation (Merlin**), two UL36 deletion viruses derived from Merlin**, or strain AD169. $\triangle$ UL36ex2 has a deletion in exon 2. (Right) the relative amount of MLKL normalized to GAPDH. Overall this data suggests that pUL36 is necessary and sufficient to degrade MLKL. 
pUL36 Protects Cells from Necroptosis. Inhibition of caspase- 8 during death receptor stimulation shifts extrinsic apoptosis toward the necroptotic pathway (29-31). The HCMV UL36encoded viral inhibitor of caspase-8 activation (vICA/pUL36) inhibits apoptosis by binding the prodomain of caspase- 8 and preventing its proteolytic activation (27). Inhibition of caspase- 8 activation by pUL36 would be predicted to promote necroptosis, but this has not been reported. We therefore sought to determine whether Merlin pUL36 additionally inhibits necroptosis by degrading MLKL. In vitro, necroptosis can be stimulated by a combination of $\mathrm{TNF} \alpha(\mathrm{T}), \mathrm{BV} 6$ (B, an IAP antagonist that sensitizes cells to TNF $\alpha$-induced cell death), and the pan-caspase inhibitor Z-VAD-fmk (Z) (Fig. 4A) (32, 33). Although previous reports have suggested that HFFFs are not susceptible to necroptosis due to limiting levels of RIP3 (34), both RIP3 and RIP1 were detectable in HFFF-TERTs by proteomics (SI Appendix, Fig. $\mathrm{S} 4 A$ ). Necroptosis was induced by $\mathrm{T}+\mathrm{B}+\mathrm{Z}$ (TBZ) in HFFF-TERTs as well as in immortalized mouse embryonic fibroblasts (MEFs), which are highly susceptible to necroptosis (35) and were therefore used as a positive control (Fig. 4B). Two inhibitors were employed to determine whether the stimulated death pathway was canonical MLKL- and RIP3-dependent necroptosis (Fig. 1). GSK'872 binds to and inhibits the RIP3 kinase domain $(21,22)$, whereas necrosulfonamide (NSA) inhibits downstream effector functions of MLKL via covalent reaction with the $\mathrm{Cys}^{86}$ residue of human but not murine MLKL $(19,23)$. NSA and GSK'872 inhibited TBZ-induced cytotoxicity in HFFF-TERTs, indicating that these cells expressed sufficient RIP3 to induce measurable canonical necroptosis (Fig. $4 B$ ).

Omoto et al. (34) previously demonstrated that pUL36 does not impact the ability of HCMV strain Towne to protect against necroptosis in fibroblasts stably transduced with RIP3. In contrast to this conclusion, we found that strain Merlin pUL36 was sufficient to inhibit necroptosis (Fig. 4C). The two other HCMVencoded inhibitors of apoptosis, pUL37 $\times 1 /$ vMIA (viral mitochondria-localized inhibitor of apoptosis) and pUL38 (37, 38), augmented MLKL-dependent necroptosis (as demonstrated by complete inhibition by NSA). However, cell death was incompletely inhibited by GSK'872, suggesting that a RIP3independent mechanism might be acting in addition (Fig. 4C). Next, untransduced, control, and UL36-expressing cell lines were treated with TB or TBZ in parallel to assess the effect of pUL36 on apoptosis and necroptosis, respectively (Fig. 4D). Other inhibitors of caspase-8, including MCMV pM36 and the compound z-VAD-fmk (Z), inhibit death receptor-stimulated apoptosis while sensitizing cells expressing sufficient levels of RIP3 and MLKL to necroptosis (29-31). Consistent with these observations, we detected no change in the overall level of cell death in untransduced cells when $\mathrm{Z}$ was added to TB (Fig. $4 \mathrm{D}$, Left leftmost bar and Right leftmost bar). In contrast, pUL36 was able to inhibit apoptotic cell death stimulated by TB, suggesting that it was not simply acting as a caspase-8 inhibitor (Fig. 4 D, Left). Furthermore, in the presence of TBZ apoptosis is potently inhibited by Z, and pUL36 inhibited necroptotic cell death (Fig. 4 D, Right). Together these data indicate that pUL36 can inhibit both apoptosis and necroptosis.

Finally, infection of HFFFs with HCMV strains Merlin and AD169 for $48 \mathrm{~h}$ prior to TBZ stimulation suggested that inhibition of MLKL-dependent necroptosis required functional pUL36. Preinfection with Merlin had a slight protective effect on necroptosis, whereas AD169 infection amplified the effect of TBZ (Fig. $4 E$ and $F$ ). Cell death induced by TBZ after infection with HCMV also appeared to have a RIP3-independent component, as GSK'872 had little inhibitory effect.

Substitution of Merlin pUL36 Cys ${ }^{131}$ Abrogates Inhibition of Necroptosis by Abolishing MLKL Binding and Degradation. Strain Merlin and strain AD169 pUL36 differ by five amino acid residues (SI Appendix, Fig. S5), including a Merlin Cys ${ }^{131} \rightarrow$ AD169 Arg $^{131}$ substitution. This single replacement abrogates inhibition of apoptosis, as pUL36 can no longer bind procaspase8 (27). Five Merlin pUL36 mutants corresponding to the five amino acid substitutions between strain Merlin and strain AD169 were constructed in order to determine which were important for degradation of MLKL. Only the C131R substitution prevented pUL36 from binding and down-regulating MLKL (Fig. $5 A$ and $B$ ). The same pUL36 mutant was also unable to protect cells from necroptosis, suggesting that this single residue plays a key functional role in inhibition of both apoptosis and necroptosis by pUL36 (Fig. $5 C$ ).

\section{Discussion}

Herpesviruses persist lifelong in infected individuals by comprehensive modulation of adaptive and innate immunity. Multiple viral proteins are deployed to target host factors for degradation, many very early during infection (10). The present study provides a systematic, searchable database that examines host protein degradation at $48 \mathrm{~h}$ of HCMV strain Merlin infection, $\sim 50$ to $66 \%$ of the way through the lytic replication cycle in HFFFs. In addition to identifying proteins degraded throughout the HCMV replication cycle, these data may be particularly useful for identifying which viral protein targets a given host factor for degradation. We defined previously the kinetics of expression of the majority of canonical HCMV proteins, which can now be compared to the kinetics of host protein degradation, from 6 to $48 \mathrm{~h}$ postinfection $(10,11)$. Other mechanisms of host protein degradation that are not inhibited by MG132 are also likely to be subverted by HCMV. However, 87 to $89 \%$ of degraded proteins were targeted to the proteasome by $24 \mathrm{~h}$ postinfection (10).

The key roles of necroptosis in protecting cell populations against virus infection are highlighted by the impressive range of known viral countermeasures. Necroptosis inhibitors are particularly widespread among herpesviruses, with MCMV and herpes simplex viruses (HSV) types 1 and 2 encoding conserved RHIMdomain containing proteins (M45, ICP6, and ICP10, respectively) which compete with host RHIM-domain adaptor proteins such as RIP1 for binding to RIP3 (39-41). The HCMV M45 ortholog pUL45 does not contain a RHIM domain and does not inhibit cell death (42). Instead, it had been suggested that HCMV targets the necroptotic pathway downstream of RHIM signaling, after RIP3-dependent phosphorylation of MLKL, although the mechanism had not been elucidated (34).

As other viral inhibitors of caspase- 8 activation have been shown to sensitize cells to programmed necrosis $(31,43)$, it has been assumed that HCMV pUL36 would have a similar effect, requiring $\mathrm{HCMV}$ to encode a separate mechanism of necroptosis inhibition in order to evade cell death completely. However, although MCMV M36 can sensitize cells to necroptosis (31), the same phenomenon has not been demonstrated for HCMV pUL36, which may not be surprising given that both proteins exhibit only $19 \%$ sequence identity (44). The understanding of why HCMV pUL36 expression can inhibit death receptor-stimulated apoptosis instead of stimulating necroptosis as per the action of other inhibitors of caspase- 8 such as z-VADfmk and MCMV M36 (29-31) may have been confounded by the use of cell lines that are not susceptible to necroptotic cell death (27). Here, we explain these observations by showing that HCMV pUL36 mediates the degradation of MLKL and inhibits necroptosis. In the presence of either UL36 expression or HCMV Merlin infection, the level of MLKL protein was decreased in cells lysed in either $6 \mathrm{M}$ guanidine, RIPA, or 2\% SDS, and subsequently rescued by the addition of MG132. Future work should confirm that high molarity guanidine and high percentage SDS do indeed fully solubilize RIPA-insoluble MLKL complexes that arise in cells undergoing necroptosis. 
A

0

Untreated
Untreated $\longrightarrow$ Lysis buffer
TNFa, BV-6 and z-VAD-fmk (TBZ)
TNFa, BV-6 and z-VAD-fmk + NSA
TNFa, BV-6 and z-VAD-fmk + GSK'872

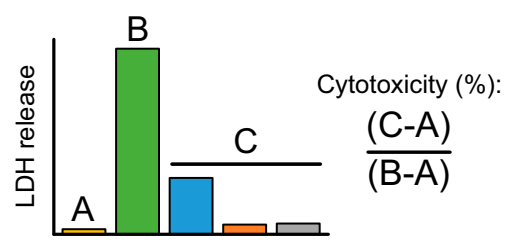

B 18

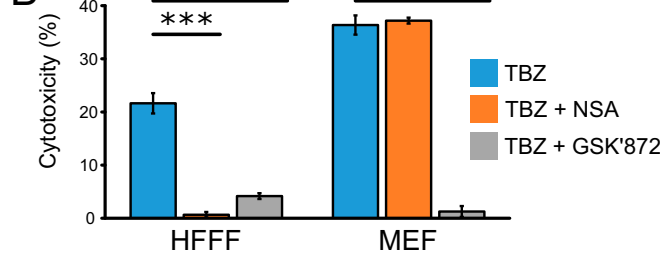

C

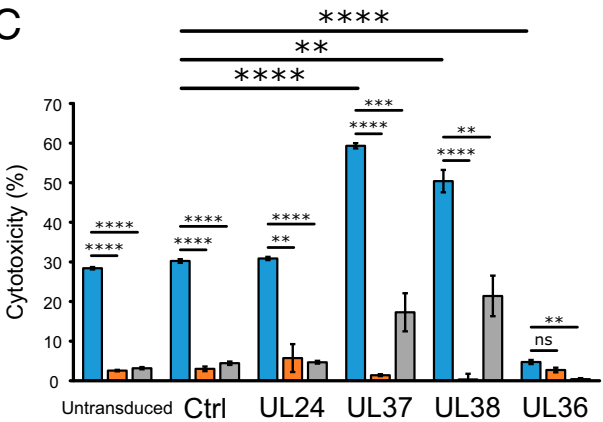

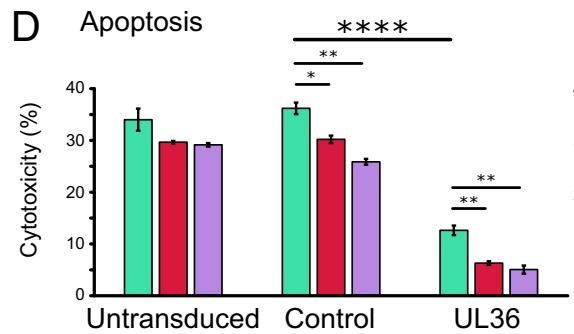

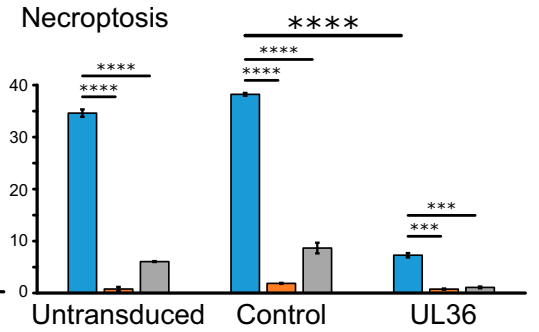

TB

$\mathrm{TB}+\mathrm{NSA}$

TB + GSK'872

TBZ

$\mathrm{TBZ}+\mathrm{NSA}$

TBZ + GSK'872
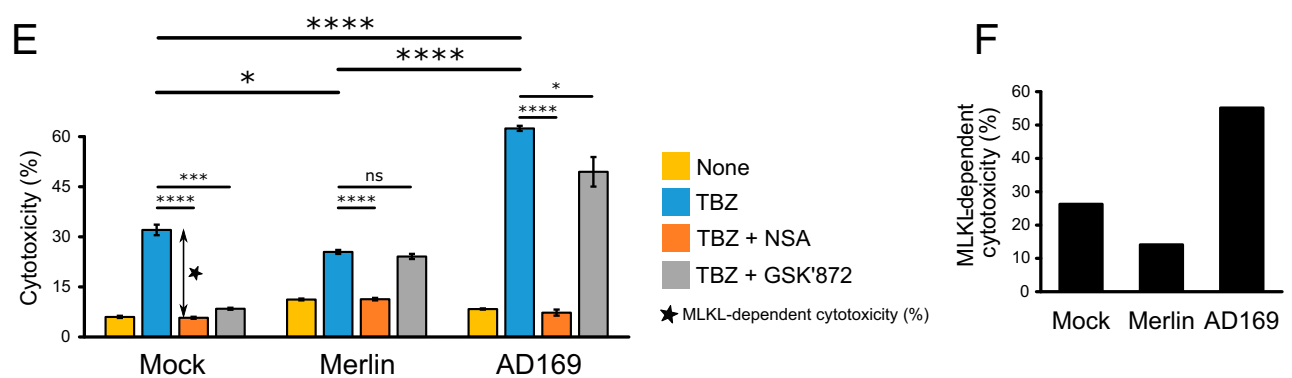

Fig. 4. Role of MLKL degradation in HCMV infection. (A) Schematic of the necroptosis assay, which was performed in biological triplicate conducted in parallel and repeated in two or three completely independent experiments. Cytotoxicity was quantified by measuring lactate dehydrogenase (LDH) release using the CytoTox 96 non-radioactive cytotoxicity assay (Promega). Cytotoxicity was calculated as a percentage of $100 \%$ lysis (from untreated cells lysed with lysis buffer) after subtraction of background LDH release from live cells. To stimulate apoptosis, cells were treated with TB instead of TBZ. (B) TBZ induces MLKL- and RIP3-dependent necroptosis, which was inhibitable by GSK' 872 in HFFF-TERTs and MEFs. NSA inhibited necroptosis in HFFF-TERTs but not MEFs, likely because NSA does not inhibit murine forms of MLKL (19). Error bars show SEM. $P$ values were estimated using a two-tailed $t$ test $(n=3)$. ***P $<0.001$, $* * * * P<0.0001$. Data are representative of three independent experiments. To dissect fully the conditions of this assay, stimulation with each of $T, B$, and $Z$ alone or in combination was examined in the presence or absence of inhibitors (SI Appendix, Fig. S4 B and C). HFFFs were insensitive to T or Z alone, TZ, or BZ. Cell death was stimulated by B alone in the absence of death receptor stimulation, an effect that has been observed in other cell types (36) (SI Appendix, Fig. S4B). The cell death initiated by BV6 was MLKL and RIP3 independent, but also caspase-8-dependent (inhibited by the addition of Z), suggesting that BV6 alone was not responsible for the necroptotic cell death observed in the TBZ condition (SI Appendix, Fig. S4 B and C). Collectively, these results suggest that only in the presence of TBZ was cell death dependent on MLKL and RIP3. (C) pUL36 is sufficient to inhibit necroptosis in stably transduced HFFF-TERTs treated with TBZ \pm inhibitors. In addition to untransduced cells, two control vectors were employed. "Ctrl" cells were transduced with a vector containing a short, randomized DNA sequence. pUL24 was included as a control HCMV tegument protein that has a similar size to pUL36 but lacks any known role in cell death. Error bars: SEM. $P$ values were estimated using a two-tailed $t$ test $(n=3) . * * P<0.01, * * * P<0.001, * * * * P<0.0001$, ns $=$ nonsignificant. Data are representative of two independent experiments. (D) pUL36 inhibits both apoptosis and necroptosis. Untransduced, control, or pUL36-expressing HFFF-TERTs were treated for $18 \mathrm{~h}$ with either TB or TBZ to stimulate apoptosis or necroptosis, respectively, in the presence or absence of NSA or GSK'872. Error bars: SEM. $P$ values were estimated using a two-tailed $t$ test $(n=3)$. ${ }^{*} P<0.05, * * P<0.01, * * * P<0.001, * * * * P<0.0001$. Data are representative of two independent experiments. (E) Functional pUL36 was required for inhibition of necroptosis, comparing $48 \mathrm{~h}$ mock, Merlin, or AD169 infection (MOI = 5) in HFFF-TERTs subsequently stimulated with TBZ \pm inhibitors for $18 \mathrm{~h}$. Baseline cytotoxicity in untreated cells was not subtracted from the other values and is shown as a separate yellow bar. Error bars: SEM. $P$ values were estimated using a two-tailed $t$ test $(n=3)$. ${ }^{*} P<0.05, * * * P<0.001, * * * * P<0.0001$, ns $=$ nonsignificant. Data are representative of two independent experiments. $(F)$ MLKL-dependent cytotoxicity was calculated from the difference between \% cytotoxicity (TBZ alone) versus (TBZ + NSA), shown by the double-headed arrow in Fig. $4 E$. 

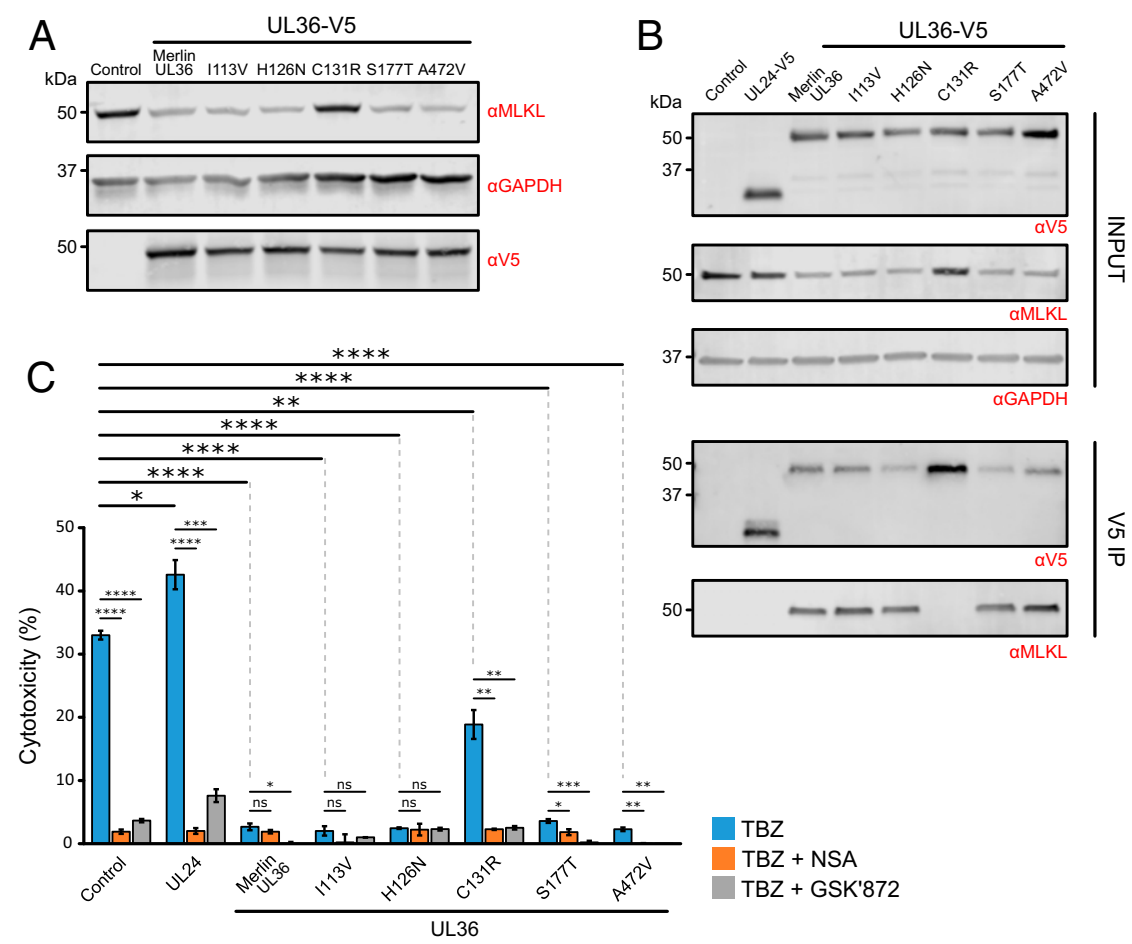

Fig. 5. Substitution of $\mathrm{Cys}^{131}$ in strain Merlin pUL36 abrogates binding to and degradation of MLKL, and inhibition of necroptosis. (A) Immunoblot showing that down-regulation of MLKL is dependent on the pUL36 Cys ${ }^{131}$ residue. HFFF-TERTs were stably transduced with the indicated C-terminally V5-tagged UL36 constructs. Individual point mutations corresponding to the five amino acid substitutions between strain Merlin and strain AD169 pUL36 are shown above the blot and detailed in SI Appendix, Fig. S5. (B) Interaction between pUL36 and MLKL is dependent on pUL36 Cys ${ }^{131}$, shown by a V5 co-IP in the cell lines described in $A$. Immunoblots were probed with anti-V5, anti-MLKL, and anti-GAPDH antibodies. (C) Percentage cytotoxicity of cell lines described in $A$ that were treated with TBZ \pm NSA or GSK'872. Error bars: SEM. $P$ values were estimated using a two-tailed $t$ test $(n=3) . * P<0.05, * * P<0.01, * * * P<0.001, * * * * P<$ 0.0001 , ns $=$ nonsignificant. Data are representative of two independent experiments. Low-level variation in sensitization to necroptosis was observed between the unmodified, control, and UL24-expressing cell lines (comparing data in this figure to Fig. 4C).

Previous studies investigating the interaction between HCMV and necroptosis have employed HFFFs stably transduced with RIP3 in order to confer susceptibility to necroptosis, which can otherwise be lost during cell propagation (34). Using these cells, the authors found that all tested HCMV strains, including AD169, were able to inhibit TNF $\alpha$-stimulated necroptosis, although strain Merlin inhibited necroptosis more potently than AD169, consistent with our results (Fig. 4E). Furthermore, comparison of a WT strain Towne (encoding pUL36 with a cysteine at position 131) with a Towne $\Delta$ UL36 mutant suggested that pUL36 was necessary for inhibition of apoptosis but not necroptosis (34). In addition to mediating cell death, RIP3 has been implicated in NF- $\mathrm{\kappa B}$ and inflammasome activation and can induce apoptosis when overexpressed $(22,45)$. It is possible that overexpression of RIP3 has off-target effects, which may explain the discrepancy between the previously published work and the present study; the experimental setting may clearly influence the outcome observed with pUL36. In addition, while the Omoto et al. study suggested that HCMV targets necroptosis downstream of MLKL phosphorylation, all MLKL peptides in our pUL36-V5 SILAC IP were unphosphorylated (Fig. $3 D$ and $S I$ Appendix, Fig. S3), suggesting that pUL36 affects the monomeric MLKL pool. However, this does not exclude the potential for additional HCMV-mediated direct or indirect mechanisms of necroptosis inhibition. Further evidence that results can be dependent on cell type and the presence of RIP3 overexpression came from use of the Towne $\Delta$ UL36 mutant to show that pUL36 can inhibit caspase-independent cell death during late stages of macrophage differentiation (46). This also suggests that pUL36mediated degradation of MLKL may occur in more than one cell type. The HFFF-TERT cell line used in the present study is susceptible to RIP3 and MLKL-dependent canonical necroptosis (Fig. 4B) and may be an invaluable resource for future studies of viral modulation of cell death.

Necroptotic activation of MLKL can influence many other cellular processes, including inflammasome activation, endosomal trafficking, extracellular vesicle generation, and autophagy (47-50). It is therefore possible that HCMV-mediated degradation of MLKL may have other consequences for viral pathogenesis, including effects on virion assembly, trafficking, and cell-to-cell spread.

Infection of HFFF-TERTs with HCMV prior to TBZ stimulation resulted in induction of a form of cell death that was not completely inhibited by GSK' 872 , which is suggestive of an RIP3-independent but MLKL-dependent mechanism (Fig. 4E). A similar but less significant effect was observed in cells expressing the HCMV apoptosis inhibitors pUL37 and pUL38 (Fig. 4C). RIP3-independent necroptosis in fibroblasts has been reported previously by others (35) but remains poorly characterized. Infection with HCMV strain AD169, which lacks a functional pUL36 protein, sensitized cells to necroptosis (Fig. $4 E$ ), in accord with the observed increase in MLKL protein upon infection with viruses deficient in functional pUL36 (Fig. $3 G$ ). This is likely to be due to IFN-mediated up-regulation of MLKL (51). Strain Merlin protein pUL36 was able to counteract the effect of necroptosis sensitization, rather than abrogating necroptosis entirely (Fig. $4 E$ ).

pUL36 can bind both procaspase-8 (27) and MLKL, facilitating potent inhibition of both apoptosis and necroptosis. Indeed, these two functions can be inhibited by the same single amino acid substitution gained during passage of HCMV in cell culture $(26,27)$. This suggests that a larger cell death complex 
may form from the caspase-8:FADD:RIP1 FADDosome/ Ripoptosome (52-54) and the RIP1:RIP3 necrosome (17), enabling an interaction between pUL36, caspase-8, and MLKL. Examination of this hypothesis, including an investigation of whether binding of pUL36 to caspase- 8 is a necessary intermediary for binding to MLKL, would be of significant interest to the field.

HCMV pUL36 and its orthologs belong to the US22 family of herpesvirus proteins, which characteristically feature a set of motifs I to IV (44). Cys ${ }^{131}$, which is found within motif III, is conserved within the primate cytomegaloviruses but is replaced by conservative substitutions in rodent cytomegalovirus orthologs that do not abrogate apoptosis inhibition. In addition, the MCMV ortholog pM36, which displays only $19 \%$ sequence similarity to HCMV pUL36 and lacks the region that includes Cys $^{131}$, is still an efficient suppressor of apoptosis (44). This suggests that the $\operatorname{Arg}^{131}$ residue of strain AD169 pUL36 may restrict cell death inhibition, as opposed to Cys ${ }^{131}$ being required for this function.

Only four drugs are currently available for treating HCMV, all exhibiting significant adverse effects and the potential of drug resistance. The identification of a potentially inhibitable interaction between a single residue of a viral antagonist and key mediators of both necroptosis and apoptosis may therefore be of substantial therapeutic significance. In addition to pUL36MLKL, other interactions involving distinct antiviral pathways could be targeted simultaneously to potently inhibit viral replication, for example between HCMV pUL145 and the recently identified restriction factor HLTF (10). Moreover, our data are likely to identify further proteins that have roles in restricting infection by HCMV or other viruses.

\section{Materials and Methods}

Extended materials and methods can be found in SI Appendix.

Viral Infections. The required volume of viral stock to achieve the MOI described in the results section was diluted in serum-free Dulbecco's Modified Eagle Medium (DMEM), mixed gently and applied to HFFF-TERTs. Mock infections were performed identically but with DMEM instead of viral stock. Time 0 was considered the time at which cells first came into contact with virus. Cells were incubated with virus for $2 \mathrm{~h}$ at $37{ }^{\circ} \mathrm{C}$ on a rocking platform, and then the medium was replaced with DMEM $+10 \%$ fetal bovine serum (FBS).

Proteomic Screen. HFFFs-TERTs were infected as described above in biological duplicate at an MOI of 10. At $36 \mathrm{hpi}, 10 \mu \mathrm{M} \mathrm{MG} 132$ or the equivalent volume of DMSO was added to the cells for the last $12 \mathrm{~h}$ of infection. Samples from the second replicate were digested and analyzed with residual samples from the 12-h degradation screen from the Nightingale et al. (10) study. Methods for whole cell lysate protein preparation and digestion, peptide labeling with tandem mass tags, HpRP fractionation, liquid chromatography-mass spectrometry, and data analysis are discussed in detail in the Nightingale et al. (10) study and recapitulated in SI Appendix.

1. M. J. Cannon, D. S. Schmid, T. B. Hyde, Review of cytomegalovirus seroprevalence and demographic characteristics associated with infection. Rev. Med. Virol. 20, 202-213 (2010).

2. T. Shenk, P. Griffiths, R. F. Pass, "Cytomegaloviruses" in Fields Virology, D. M. Knipe, P. M. Howley, Eds. (Lippincott Williams \& Wilkins, Philadelphia, PA, 6th Ed., 2013), pp. 2702-2772.

3. P. Reinke, S. Prösch, F. Kern, H. D. Volk, Mechanisms of human cytomegalovirus (HCMV) (re)activation and its impact on organ transplant patients. Transpl. Infect. Dis. 1, 157-164 (1999).

4. W. G. Nichols, L. Corey, T. Gooley, C. Davis, M. Boeckh, High risk of death due to bacterial and fungal infection among cytomegalovirus (CMV)-seronegative recipients of stem cell transplants from seropositive donors: Evidence for indirect effects of primary CMV infection. J. Infect. Dis. 185, 273-282 (2002).

5. W. J. Britt, Congenital human cytomegalovirus infection and the enigma of maternal immunity. J. Virol. 91, e02392-16 (2017).

6. B. H. Tan, Cytomegalovirus treatment. Curr. Treat. Options Infect. Dis. 6, 256-270 (2014).

7. R. Nathans et al., Small-molecule inhibition of HIV-1 Vif. Nat. Biotechnol. 26, 1187-1192 (2008)
Immunoblotting. Cells were lysed, sonicated, and clarified by centrifugation, and protein concentration was measured using a bicinchoninic acid (BCA) assay. Samples were denatured and reduced, and then the proteins were separated by sodium dodecyl sulfate (SDS) polyacrylamide gel electrophoresis (PAGE), transferred to a polyvinylidene difluoride (PVDF) membrane $(0.45 \mu \mathrm{m}$ pore), and probed using the primary and secondary antibodies detailed in $S$ Appendix. Fluorescent signals were detected using the Odyssey CLx Imaging System (LI-COR), and images were processed and quantified using Image Studio Lite V5.2 (LI-COR).

Plasmid Construction and Transduction. Lentiviral expression vectors encoding MLKL-HA, the V5-tagged viral proteins pUL36 and pUL133-pUL150A and controls, were synthesized by PCR amplification of the genes and cloning them into Gateway vectors (55). V5-tagged UL36 point mutants were generated by PCR site-directed mutagenesis. The primers and templates used are described in SI Appendix. Stable cell lines were generated by transduction with lentiviruses produced via the transfection of HEK293T cells with lentiviral expression vectors and helper plasmids.

Immunoprecipitation. Cells were harvested in lysis buffer, tumbled on a rotator and then clarified by centrifugation and filtration. After incubation with immobilized mouse monoclonal anti-V5 or anti-HA agarose resin, samples were washed and then subjected either to immunoblotting or mass spectrometry (SI Appendix).

Cell Death Assays. For assays in the absence of infection, 96-well plates were seeded with HFFF-TERTs or immortalized MEFs and incubated for $24 \mathrm{~h}$. Cells were incubated for $18 \mathrm{~h}$ with $30 \mathrm{ng} / \mathrm{mL}$ TNF $\alpha, 5 \mu \mathrm{M}$ BV-6, and/or $25 \mu \mathrm{M}$ z-VAD-fmk in the presence or absence of $0.5 \mu \mathrm{M}$ necrosulfonamide (NSA) or $1.5 \mu \mathrm{M}$ GSK' 872 , or DMSO alone (control). Half of the control cells were lysed to measure lactate dehydrogenase (LDH) release from maximally lysed cells. The other half were used to measure background LDH release from live, untreated cells. LDH release was measured using the CytoTox 96 NonRadioactive Cytotoxicity Assay. Average absorbance values derived from culture medium alone were subtracted from each absorbance value from experimental wells. For assays in the presence of infection, HFFF-TERTs were seeded into 96-well plates, incubated for $24 \mathrm{~h}$, and infected with HCMV $(\mathrm{MOI}=5)$. The medium was changed $48 \mathrm{~h}$ after infection to stimulate necroptosis as described above.

Data and Materials Availability Statement. The mass spectrometry proteomics data have been deposited to the ProteomeXchange Consortium (www proteomexchange.org/) via the PRIDE partner repository (56) with the dataset identifier PXD017279 (57). All materials described in this manuscript and any further details of protocols employed, can be obtained on request from the corresponding author by email to mpw1001@cam.ac.uk.

ACKNOWLEDGMENTS. We are grateful to Prof. Steve Gygi for providing access to the "MassPike" software pipeline for quantitative proteomics. This work was supported by an Medical Research Council (MRC) studentship (MR/ N013433/1) to A.F.-E., a Wellcome Trust Senior Clinical Research Fellowship (108070/Z/15/Z) to M.P.W., an MRC Project Grant (MR/P001602/1) to R.J.S., and an MRC Programme Grant (MC_UU_12014/3) to A.J.D. This study was additionally supported by the Cambridge Biomedical Research Centre, UK.

8. S. Cen et al., Small molecular compounds inhibit HIV-1 replication through specifically stabilizing APOBEC3G. J. Biol. Chem. 285, 16546-16552 (2010).

9. N. Stern-Ginossar et al., Decoding human cytomegalovirus. Science 338, 1088-1093 (2012).

10. K. Nightingale et al., High-definition analysis of host protein stability during human cytomegalovirus infection reveals antiviral factors and viral evasion mechanisms. Cell Host Microbe 24, 447-460.e11 (2018).

11. M. P. Weekes et al., Quantitative temporal viromics: An approach to investigate hostpathogen interaction. Cell 157, 1460-1472 (2014).

12. A. Halenius, C. Gerke, H. Hengel, Classical and non-classical MHC I molecule manipulation by human cytomegalovirus: So many targets-but how many arrows in the quiver? Cell. Mol. Immunol. 12, 139-153 (2015).

13. K. Viswanathan, K. Früh, V. Defilippis, Viral hijacking of the host ubiquitin system to evade interferon responses. Curr. Opin. Microbiol. 13, 517-523 (2010).

14. Q. Tang, P. Wu, H. Chen, G. Li, Pleiotropic roles of the ubiquitin-proteasome system during viral propagation. Life Sci. 207, 350-354 (2018)

15. W. J. Kaiser, J. W. Upton, E. S. Mocarski, Viral modulation of programmed necrosis. Curr. Opin. Virol. 3, 296-306 (2013) 
16. R. C. Taylor, S. P. Cullen, S. J. Martin, Apoptosis: Controlled demolition at the cellular level. Nat. Rev. Mol. Cell Biol. 9, 231-241 (2008).

17. J. Zhang, Y. Yang, W. He, L. Sun, Necrosome core machinery: MLKL. Cell. Mol. Life Sci. 73, 2153-2163 (2016)

18. X. Chen et al., Translocation of mixed lineage kinase domain-like protein to plasma membrane leads to necrotic cell death. Cell Res. 24, 105-121 (2014).

19. L. Sun et al., Mixed lineage kinase domain-like protein mediates necrosis signaling downstream of RIP3 kinase. Cell 148, 213-227 (2012)

20. E. J. Petrie, P. E. Czabotar, J. M. Murphy, The structural basis of necroptotic cell death signaling. Trends Biochem. Sci. 44, 53-63 (2019).

21. W. J. Kaiser et al., Toll-like receptor 3-mediated necrosis via TRIF, RIP3, and MLKL. J. Biol. Chem. 288, 31268-31279 (2013).

22. P. Mandal et al., RIP3 induces apoptosis independent of pronecrotic kinase activity Mol. Cell 56, 481-495 (2014).

23. S. Liu et al., MLKL forms disulfide bond-dependent amyloid-like polymers to induce necroptosis. Proc. Natl. Acad. Sci. U.S.A. 114, E7450-E7459 (2017).

24. J. Cox, M. Mann, MaxQuant enables high peptide identification rates, individualized p.p.b.-range mass accuracies and proteome-wide protein quantification. Nat. Bio technol. 26, 1367-1372 (2008).

25. E. Clark, D. H. Spector, Studies on the contribution of human cytomegalovirus UL21a and UL97 to viral growth and inactivation of the Anaphase-Promoting Complex Cyclosome (APC/C) E3 ubiquitin ligase reveal a unique cellular mechanism for downmodulation of the APC/C subunits APC1, APC4, and APC5. J. Virol. 89, 6928-6939 (2015).

26. A. J. Bradley et al., High-throughput sequence analysis of variants of human cytomegalovirus strains Towne and AD169. J. Gen. Virol. 90, 2375-2380 (2009).

27. A. Skaletskaya et al., A cytomegalovirus-encoded inhibitor of apoptosis that suppresses caspase-8 activation. Proc. Natl. Acad. Sci. U.S.A. 98, 7829-7834 (2001).

28. L. V. Nobre et al., Human cytomegalovirus interactome analysis identifies degradation hubs, domain associations and viral protein functions. eLife 8, e49894 (2019).

29. N. Holler et al., Fas triggers an alternative, caspase-8-independent cell death pathway using the kinase RIP as effector molecule. Nat. Immunol. 1, 489-495 (2000).

30. S. McComb et al., Cathepsins limit macrophage necroptosis through cleavage of Rip1 kinase. J. Immunol. 192, 5671-5678 (2014).

31. W. J. Kaiser et al., RIP3 mediates the embryonic lethality of caspase-8-deficient mice. Nature 471, 368-372 (2011)

32. M. El-Mesery, M. E. Shaker, A. Elgaml, The SMAC mimetic BV6 induces cell death and sensitizes different cell lines to TNF- $\alpha$ and TRAIL-induced apoptosis. Exp. Biol. Med. (Maywood) 241, 2015-2022 (2016)

33. N. M. de Vasconcelos, N. Van Opdenbosch, H. Van Gorp, E. Parthoens, M. Lamkanfi, Single-cell analysis of pyroptosis dynamics reveals conserved GSDMD-mediated subcellular events that precede plasma membrane rupture. Cell Death Differ. 26, 146-161 (2019).

34. S. Omoto et al., Suppression of RIP3-dependent necroptosis by human cytomegalovirus. J. Biol. Chem. 290, 11635-11648 (2015).

35. D.-W. Zhang et al., Multiple death pathways in TNF-treated fibroblasts: RIP3- and RIP1-dependent and independent routes. Cell Res. 21, 368-371 (2011).

36. D. E. Christofferson, Y. Li, J. Yuan, Control of life-or-death decisions by RIP1 kinase Annu. Rev. Physiol. 76, 129-150 (2014).

37. C. E. Andoniou, M. A. Degli-Esposti, Insights into the mechanisms of CMV-mediated interference with cellular apoptosis. Immunol. Cell Biol. 84, 99-106 (2006).

38. S. Terhune et al., Human cytomegalovirus UL38 protein blocks apoptosis. J. Virol. 81, 3109-3123 (2007)
39. H. Guo, W. J. Kaiser, E. S. Mocarski, Manipulation of apoptosis and necroptosis signaling by herpesviruses. Med. Microbiol. Immunol. 204, 439-448 (2015)

40. J. W. Upton, W. J. Kaiser, E. S. Mocarski, DAI/ZBP1/DLM-1 complexes with RIP3 to mediate virus-induced programmed necrosis that is targeted by murine cytomegalovirus vIRA. Cell Host Microbe 11, 290-297 (2012).

41. H. Guo et al., Herpes simplex virus suppresses necroptosis in human cells. Cell Host Microbe 17, 243-251 (2015)

42. M. Patrone et al., The human cytomegalovirus UL45 gene product is a late, virionassociated protein and influences virus growth at low multiplicities of infection. J. Gen. Virol. 84, 3359-3370 (2003).

43. M. Li, A. A. Beg, Induction of necrotic-like cell death by tumor necrosis factor alpha and caspase inhibitors: Novel mechanism for killing virus-infected cells. J. Virol. 74, 7470-7477 (2000).

44. A. L. McCormick, A. Skaletskaya, P. A. Barry, E. S. Mocarski, V. S. Goldmacher, Differential function and expression of the viral inhibitor of caspase 8-induced apoptosis (vICA) and the viral mitochondria-localized inhibitor of apoptosis (VMIA) cell death suppressors conserved in primate and rodent cytomegaloviruses. Virology 316, 221-233 (2003)

45. K. Moriwaki, F. K.-M. Chan, The inflammatory signal adaptor RIPK3: Functions beyond necroptosis. Int. Rev. Cell Mol. Biol. 328, 253-275 (2017)

46. A. L. McCormick, L. Roback, D. Livingston-Rosanoff, C. St Clair, The human cytomegalovirus UL36 gene controls caspase-dependent and -independent cell death programs activated by infection of monocytes differentiating to macrophages. J. Virol. 84, 5108-5123 (2010).

47. S. Kang et al., Caspase-8 scaffolding function and MLKL regulate NLRP3 inflammasome activation downstream of TLR3. Nat. Commun. 6, 7515 (2015).

48. S. A. Conos et al., Active MLKL triggers the NLRP3 inflammasome in a cell-intrinsic manner. Proc. Natl. Acad. Sci. U.S.A. 114, E961-E969 (2017).

49. S. Yoon, A. Kovalenko, K. Bogdanov, D. Wallach, MLKL, the protein that mediates necroptosis, also regulates endosomal trafficking and extracellular vesicle generation. Immunity 47, 51-65.e7 (2017).

50. D. Frank, D. L. Vaux, J. M. Murphy, J. E. Vince, L. M. Lindqvist, Activated MLKL attenuates autophagy following its translocation to intracellular membranes. J. Cell Sci. 132, jcs220996 (2019).

51. I. Rusinova et al., Interferome v2.0: An updated database of annotated interferonregulated genes. Nucleic Acids Res. 41, D1040-D1046 (2013).

52. C. M. Henry, S. J. Martin, Caspase-8 acts in a non-enzymatic role as a scaffold for assembly of a pro-inflammatory "FADDosome" complex upon TRAIL stimulation. Mol. Cell 65, 715-729.e5 (2017).

53. M. Feoktistova et al., cIAPs block Ripoptosome formation, a RIP1/caspase-8 containing intracellular cell death complex differentially regulated by cFLIP isoforms. Mol. Cell 43, 449-463 (2011)

54. T. Tenev et al., The Ripoptosome, a signaling platform that assembles in response to genotoxic stress and loss of IAPs. Mol. Cell 43, 432-448 (2011).

55. J. L. Hartley, G. F. Temple, M. A. Brasch, DNA cloning using in vitro site-specific recombination. Genome Res. 10, 1788-1795 (2000).

56. J. A. Vizcaíno et al., 2016 update of the PRIDE database and its related tools. Nucleic Acids Res. 44, D447-D456 (2016)

57. A. Fletcher-Etherington et al, Human cytomegalovirus protein pUL36: A dual cell death pathway inhibitor. PRIDE. https://www.ebi.ac.uk/pride/archive/projects/PXD017279. Deposited 24 January 2020. 